\title{
CORRECTION
}

\section{Correction to: Simple current control without grid voltage sensor for traction solid-state transformer}

\author{
Chun-Gi Yun ${ }^{1}$ S Seunghoon Baek ${ }^{1} \cdot$ Hanyoung $\mathrm{Bu}^{1} \cdot$ Younghoon $\mathrm{Cho}^{1}{ }^{1} \cdot$ Jin-Hyuk Park ${ }^{2} \cdot$ Myung-Yong Kim $^{2}$
}

Published online: 17 May 2021

(c) The Korean Institute of Power Electronics 2021

\section{Correction to: \\ Journal of Power Electronics (2021) 21:703-712 \\ https://doi.org/10.1007/s43236-020-00214-4}

Due to an unfortunate oversight the affiliations have been interchanged. They should read:

${ }^{1}$ Department of Electrical and Electronics Engineering, Konkuk University, Seoul, Korea.

${ }^{2}$ Korea Railroad Research Institute, Uiwang, Korea.

The original article can be found online at https://doi.org/10.1007/ s43236-020-00214-4.

Younghoon Cho

yhcho98@konkuk.ac.kr

1 Department of Electrical and Electronics Engineering,

Konkuk University, Seoul, Korea

2 Korea Railroad Research Institute, Uiwang, Korea 